\title{
PA-031 SPATIAL-TEMPORAL DYNAMICS IN HETEROGENEITY OF MALARIA INFECTION IN A SETTING WITH SEASONAL TRANSMISSION: A LONGITUDINAL STUDY IN THE GAMBIA
}

Julia Mwesigwa, ${ }^{1}$ Jane Achan, ${ }^{1}$ Musa Jawara, ${ }^{1}$ Gian Ditanna, ${ }^{2}$ Archibald Worwui, ${ }^{1}$ Muna Affara, ${ }^{1}$ Jean-Pierre Geertruyden, ${ }^{3}$ Umberto D'Alessandro ${ }^{4}$. ${ }^{1} M R C$, The Gambia; ${ }^{2}$ QMUL, United Kingdom; ${ }^{3}$ University of Antwerp, Belgium; ${ }^{4}$ LSHTM, United Kingdom

10.1136/bmjgh-2016-000260.67

Background The reduction in the malaria burden previously reported in The Gambia is largely due to the successful scaling up of control interventions. Understanding the current dynamics of malaria transmission in a context of high coverage of control interventions is critical to inform pre-elimination efforts.

Methods A cohort study was conducted in 12 villages across the country during the 2013 transmission season. Enrolled residents aged over 6 months old had a blood sample collected monthly for molecular analysis (PCR) and microscopy. Clinical malaria cases were captured by passive detection. Mosquito abundance and species distribution were determined by collections with CDC light traps, human landing catches.

Results A cohort of 4235 participants with a median age of 13 years (IQR 5, 28) was followed up. Long Lasting Insecticidal Nets coverage was $71.6 \%$ (2774/3876). The incidence rate of Plasmodium falciparum parasitaemia infection was 1.1 episodes per person-year (95\% CI: 0.8-1.2). P. falciparum transmission was heterogeneous with low rates in the western region 0.47 episodes p-pyear (0.41-0.56) and highest in the eastern region 2.8 episodes per person-year (95\% CI: 2.6-3.1). The peak mosquito densities were in September preceding peak $P$. falciparum incidence in December. Anopheles (An.) Gambiae $S$ form and $A n$. arabiensis were the predominant species in all the regions except the central and lower river regions where $A n$. gambiae M form and An. melas were the predominant species. The risk of clinical malaria during the season was higher among individuals living with asymptomatic malaria at the start of the season; (Western region $\mathrm{HR}=3.9,95 \% \mathrm{CI}: 2.1-7.5$ ) and eastern region (HR=1.5, 95\% CI: 1.1-2.1).

Conclusions In The Gambia, malaria transmission is seasonal and heterogeneous across the country, with clustering of infection and disease at household level, suggesting the need for targeted interventions. 